\title{
Painting a More Colorful Picture: A Review of Recently Proposed Vitiligo Treatments
}

\section{Georgina M Ferzli*}

SUNY Downstate Medical Center, USA

\begin{abstract}
Vitiligo is a depigmentation disorder of the skin with profound physical and psychological effects. Several techniques have been developed over the years to treat this disorder, including combinations of topical treatments, oral medications, light therapies, and laser treatments. The current mainstay for treatment is narrow-band ultra-violet B (NB-UVB), topical calcineurin inhibitors, and topical corticosteroids. Still, many patients today continue to suffer from the effects of this disorder. This paper aims to present new, promising treatments for vitiligo that has been presented in the literature of late.
\end{abstract}

Keywords: Vitiligo; Topical treatments; Narrow-Band Ultra-Violet B (NB-UVB); Laser treatments

\section{Introduction}

Vitiligo is a hypomelanotic disorder of the skin that results from loss of functional melanocytes in the skin [1]. The disorder affects people of all ages, races, and sexes, with a prevalence of $1-2 \%$, and half of people with vitiligo have disease onset before the age of 20 years [1]. Vitiligo can lead to stress and embarrassment, particularly in darker-skinned individuals where the loss of pigment leads to a stark contrast in color [2,3]. To date, there is no universally accepted safe and efficacious treatment for vitiligo. Relapse is very common and complete repigmentation is rare [4].

The main histopathological finding in vitiligo is the total absence of functioning melanocytes in the lesions. The main inflammatory cells most commonly found on the edges of these lesions are CD4+ and CD8+ T lymphocytes [5]. Based on this principle, commonly used treatment strategies have focused on control of the autoimmune damage as well as stimulation of melanocyte migration from the unaffected edges of the lesions to the affected skin [5]. Currently, treatment for vitiligo can be categorized into three basic groups: pharmacological (both topical and systemic) treatment, physical treatment, and surgical treatment [5]. Examples include topical and systemic corticosteroids, topical calcineurin inhibitors, NVUVB therapy, phototherapy with UVA and psoralens (PUVA), and surgical transplantation of melanocytes [5]. Again, it is well known that all of these treatments have major setbacks and that complete repigmentation is uncommon.

Recently, the literature has suggested various novel treatments for vitiligo. This paper will aim to presentseveral new treatments proposed in the literature over the past year.

\section{Discussion}

\section{Intralesional corticosteroids}

Topical steroids are frequently used in the treatment of vitiligo. Literature has shown varying levels of efficacy with topical steroids and high rates of relapse upon tapering the topical steroids [6]. Topical steroids also carry the risk of atrophy, telangectasias, and striae, as well as systemic side effects, and are therefore best used in localized areas [6]. Interestingly, a meta-analysis in 1998 showed that class 3 topical steroids were most effective in treating localized vitiligo compared to class 4 topical steroids, while patients using class 4 drugs also demonstrated higher rates of atrophy $[5,7]$. This difference in efficacy may suggest that achieving a particularmilieu for normal cell function is the ultimate goal.
A treatment modality not yet commonly used for vitiligo is intralesional corticosteroid injections [8]. For the first time in 30 years, new literature shows that intralesional corticosteroid injections are a safe and very effective treatment for vitiligo [8-11]. Wang et al. followed nine adult females with vitiligo from 2009 to 2013 and treated them with intralesional triamcinolone injections to vitiligo patches every four to six weeks [8]. Of these patients, six were treatment-resistant and three were treatment-naïve. Those who were being treated with inadequate NBUVB and/or topical corticosteroids had the medications continued. Remarkably, all nine patients responded to the intralesional corticosteroid injections with $80-90 \%$ repigmentation. The average duration of treatment was four months, with the longest duration being only seven months [8]. The article demonstrates that some patients repigmented up to $75 \%$ from just one treatment. Average start time to repigmentation was one month, and the earliest repigmentation was seen at three weeks [8]. The untreated vitiliginous patches did not show any repigmentation. This study was also remarkable in that most patients maintained their pigmentation for years after cessation of injections. Furthermore, in the patients that did lose pigment, no more than $15 \%$ depigmentation was seen [8]. At this point, one may ask about the side effects of the injections. Astoundingly, complications in this study were minimal, with skin atrophy seen in only one patient. Two of the women showed menstrual irregularities, but resumed normal cycles after cessation of treatment [8]. This study demonstrates that more research must be conducted on a larger scale to see if intralesional corticosteroid injections should be used more often in the treatment of vitiligo.

\section{A famelanotide and narrowband UVB phototherapy}

A recent study by Lim et al. proposed utilizing the body's cutaneous melanocortin system to help treat vitiligo. This system contains $\alpha$-melanocyte-stimulating hormone ( $\alpha$-MSH), an important protein that stimulates melanogenesis and melanocyte proliferation [12]. Lim et al. used afamelanotide, a potent synthetic analogue of $\alpha-\mathrm{MSH}$ in a controlled-release formulation, in combination with NBUVB

*Corresponding author: Georgina M Ferzli, SUNY Downstate Medical Center Brooklyn, NY 11203, USA, Tel: 631-864-6647; E-mail: georginamarie@gmail.com

Received: October 20, 2014; Accepted: October 22, 2014; Published: October 27,2014

Citation: Ferzli GM (2014) Painting a More Colorful Picture: A Review of Recently Proposed Vitiligo Treatments. Pigmentary Disorders 1:143. doi: 10.4172/ JPD. 1000143

Copyright: () 2014 Ferzli GM. The terms of the Creative Commons Attribution License, which permits unrestricted use, distribution, and reproduction in any medium, provided the original author and source are credited. 
phototherapy to conduct their study $[11,13]$. The study evaluated the efficacy and safety of combination therapy consisting of afamelanotide and NBUVB phototherapy for treating generalized vitiligo. The combination treatment was evaluated against NBUVB alone, with scoring based on response on the Vitiligo Area Scoring Index and Vitiligo European Task Force scoring system [11]. Patients in the study had vitiligo that involved $15-50 \%$ of total body surface area, with stable or slowly progressive vitiligo for three months. The treatment group received monthly subcutaneous injections of afamelanotide for four months, while the control group received only NB-UVB for the duration of the study [12]. Results showed that the combination therapy group displayed superior response compared to the NB-UVB monotherapy group, both at day 56 and at day 168 [11]. The combination therapy group showed an especially higher percentage of patients achieve repigmentation in the face and upper extremities, and at earlier times (41 days vs. 61 days for the face, and 46 days vs. 69 days for the upper extremities) [11]. Adverse effects included erythema in both groups, and minor infections and nausea in the combination group [11]. A combination of a famelanotide and NB-UVB phototherapy resulted in clinically apparent, statistically significant superior and faster repigmentation compared with NB-UVB monotherapy [11]. These findings suggest that perhaps focusing on biologic analogs in order to treat vitiligo would be beneficial going forward, and calls for more research in this area.

\section{Antioxidative and anti-inflammatory supplementation}

The role of antioxidants and anti-inflammatory compounds has recently come into the spotlight for vitiligo treatment. In the past year, several compounds have been studied, including epigallocatechin-3gallate (ECGC), Polypodium leucotomos, and Phyllanthus emblica [1416]. One study by Colucci et al. focuses on three compounds: Phyllanthus emblica, vitamin E, and carotenoids. These three compounds show antioxidative, anti-inflammatory, and repigmenting effects [16]. In this study, 65patients were given an oral supplement containing $P$. emblica $(100 \mathrm{mg})$, vitamin E (4.7 $\mathrm{mg})$, and carotenoids $(10 \mathrm{mg})$ three times daily for six months. Another group of 65 patients was not given any oral supplement. Both groups were treating simultaneously with comparable topical therapy and/or phototherapy. At six-month followup, a significantly higher percentage of patients in the supplementation group showed repigmentation on the head/neck regions and on the trunk [16]. Furthermore, the number of patients who showed no repigmentation in head/neck, trunk, and limbs was significantly higher in the group that did not receive the antioxidants. This control group also showed higher signs of inflammation; faster growing lesions, more erythema, and higher percentage of overall worsening of disease [16]. These results suggest benefit in supplementing with antioxidants in order to increase effectiveness of other vitiligo treatments [16].

Another antioxidant that has received much attention in the antiaging literature is epigallocathecin-3-gallate (EGCG) [14]. It is one of the main chemical constituents of green tea, with anti-inflammatory, antioxidant, and immunomodulatory properties. Recently, Zhu et al. proposed the idea of using this chemical to supplement vitiligo treatment. Unlike the studies discussed above, this study was performed in mice [14]. The study demonstrated that EGCG supplementation delayed the time of depigmentation, reduced the prevalence of depigmentation, and decreased the area of depigmentation. Furthermore, examination using reflectance confocal microscopy showed that the depigmented skin that was treated with ECGC displayed increased numbers of epidermal melanocytes. Histologic examination showed decreased peri-lesional accumulation of CD8+ T cells. Moreover, levels of tumor necrosis factor (TNF)- $\alpha$, interferon (IFN)- $\gamma$ and interleukin (IL)- 6 were significantly decreased after administration of EGCG compared with the control group [14]. These results suggest that EGCG may have protective effects against vitiligo, and that it could contribute to suppression of activation of CD8+ T cells and inflammatory mediators [14].

Based on these results, Zhu et al. conducted further studies on these mice by investigating the gene-expression profile of this model in relation to EGCG [14]. Using whole genome oligo-microarray assay, 1264 down-regulated genes and 1332 up-regulated genes were recorded in the EGCG group compared with the model group, and selected genes were validated by real-time polymerase chain reaction [14]. Remarkably, the genetic study demonstrated that EGCG administration was significantly associated with a decreased risk of vitiligo [14]. Therefore, based on these results, EGCG could be considered a new preventive agent against vitiligo in the clinical setting [14]. This model demonstrates the need for further research into antioxidants and their effects on vitiligo, especially given their qualities of benign nature, easy accessibility, easy administration, and cost-efficiency.

\section{T-regulatory cells and control of vitiligo}

As discussed earlier, immunology likely plays a role in the pathogenesis of vitiligo. Dwivedi et al. recently published an excellent review of the various immunotherapies currently being studied for treatment of vitiligo [17]. Dependent on the fact that regulatory T-cells (Tregs) are critical to the development of self-tolerance, they can be considered a major focus in studying the autoimmune pathogenesis of vitiligo [17]. In this review, Dwivedi discusses the autoimmune pathogenesis of vitiligo, and goes on to discuss current therapeutic options being studied based on these principles. An interesting animal study in particular was performed by Catterjee et al. using transgenic mice. The mice carry T-cells with human leukocyte antigen (HLA)A2-restricted human tyrosinase peptide-reactive T-cell receptor and develop vitiligo from an early age $[17,18]$. Tregs were adoptively transferred into these mice and were found to induce a lasting remission of vitiligo when transferred at the onset of the disease, and also when transferred during established disease [18]. The study showed that reduced regulatory responses were critical to the development of vitiligo in disease-prone mice, and suggested that an increase in the Treg cell population might benefit vitiligo patients with active disease [18]. This was the first animal study of its kind $[17,18]$. These fascinating results, as well as other studies reviewed by Dwivedi et al., call for further study of Tregs and their role in the therapeutics of vitiligo.

\section{Conclusions}

Though vitiligo still remains a difficult disease to treat, recent progress in its research has presented a rainbow of various therapeutic options for the future. With continued focus on immunology, genetics, and melanogenesis, perhaps scientific advances will bring more hope and improved quality of life to vitiligo patients going forward.

\section{References}

1. Taïeb A, Picardo M (2010) Epidemiology, definitions and classification. Vitiligo, Springer-Verlag, Berlin: 13-24.

2. Ongenae K, Beelaert L, van Geel N, Naeyaert JM (2006) Psychosocial effects of vitiligo. J Eur Acad Dermatol Venereol 20: 1-8

3. Taïeb A, Picardo M (2010) Management overview. Vitiligo, Springer-Verlag Berlin: 319-323.

4. Ongenae K, Dierckxsens L, Brochez L, van Geel N, Naeyaert JM (2005) Quality of life and stigmatization profile in a cohort of vitiligo patients and effect of the use of camouflage. Dermatology 210: 279-285.

5. Faria AR, Tarlé RG, Dellatorre G, Mira MT, de Castro CC (2014) Vitiligo--Part 2--classification, histopathology and treatment. An Bras Dermatol 89: 784-790. 
Citation: Ferzli GM (2014) Painting a More Colorful Picture: A Review of Recently Proposed Vitiligo Treatments. Pigmentary Disorders 1:143. doi: 10.4172/JPD.1000143

6. Lotti T, Berti S, Moretti S (2009) Vitiligo therapy. Expert Opin Pharmacother 10 2779-2785.

7. Njoo MD, Spuls PI, Bos JD, Westerhof W, Bossuyt PM (1998) Nonsurgical repigmentation therapies in vitiligo. Meta-analysis of the literature. Arch Dermatol 134: 1532-1540.

8. Wang E, Koo J, Levy E (2014) Intralesional corticosteroid injections for vitiligo: a new therapeutic option. J Am Acad Dermatol 71: 391-393.

9. Kandil E (1970) Treatment of localized vitiligo with intradermal injections of triamcinolone acetonide. Dermatologica 140: 195-206.

10. Vasistha LK, Singh G (1979) Vitiligo and intralesional steroids. Indian J Med Res 69: 308-311.

11. Lim HW, Grimes PE, Agbai O, Hamzavi I, Henderson M, et al. (2014) Afamelanotide and Narrowband UV-B Phototherapy for the Treatment of Vitiligo: A Randomized Multicenter Trial. JAMA Dermatol

12. Abdel-Malek Z, Scott MC, Suzuki I, Tada A, Im S, et al. (2000) The melanocortin-1 receptor is a key regulator of human cutaneous pigmentation. Pigment Cell Res 13 Suppl 8: 156-162.
13. Minder EI (2010) Afamelanotide, an agonistic analog of Ît-melanocytestimulating hormone, in dermal phototoxicity of erythropoietic protoporphyria. Expert Opin Investig Drugs 19: 1591-1602.

14. Zhu Y, Wang S, Lin F, Li Q, Xu A (2014) The therapeutic effects of EGCG on vitiligo. Fitoterapia .

15. Nestor M, Bucay V, Callender V, Cohen JL, Sadick N, et al. (2014) Polypodium leucotomos as an Adjunct Treatment of Pigmentary Disorders. J Clin Aesthet Dermatol 7: 13-17.

16. Colucci R, Dragoni F, Conti R, Pisaneschi L, Lazzeri L, et al. (2014) Evaluation of an oral supplement containing Phyllanthus emblica fruit extracts, vitamin $\mathrm{E}$, and carotenoids in vitiligo treatment. Dermatol Ther

17. Dwivedi M, Helen Kemp E, Laddha NC, Mansuri MS, Weetman AP, et al. (2014) Regulatory T cells in vitiligo: Implications for pathogenesis and therapeutics. Autoimmun Rev .

18. Chatterjee S, Eby JM, Al-Khami AA, Soloshchenko M, Kang HK, et al. (2014) A quantitative increase in regulatory $T$ cells controls development of vitiligo. $J$ Invest Dermatol 134: 1285-1294. 